\title{
CHARACTERIZATION OF FAILURE BEHAVIOR OF ADVANCED COMPOSITES USING OFF-AXIS FLEXURAL STRENGTH TESTS
}

\author{
R. S. Raghava
}

Polymer \& Composite Research, Westinghouse R\&D Center, Pittsburgh, PA 15235, USA

ABSTRACT

This paper discusses failure behavior of graphite/epoxy and Kevlar/epoxy advanced composites. Flexural strengths of unidirectional and cross-ply composites were measured by taking off-axis flexural specimens at regular intervals.

test. Results are compared with several failure criteria existing in the literature.

\section{KEYWORDS}

Advanced composites; failure; off-axis flexural strength; graphite-epoxy; Kev1ar-epoxy.

INTRODUCTION

Composite materials are finding ever-increasing use in the structural appli cations. It is essential to establish a general failure criterion to act as a guide for the design engineer. An essential requirement of a failure criterion is that it should be general but it should be simple to use. Ther are two alternate approaches: a criterion based on the micromechanics of composites describing failure behavior at the micro level; another approach is to examine failure behavior of composites at the macro level. The first suggested approach is very general but it is cumbersome, and unwieldy to use in design calculations. In the second approach, a phenomenological criterion describing a three dimensional stress state is assumed. A convenient representation of such a criterion is in terms of polynominals. It is then essential to establish coefficients of the polynomial experimentally. The coefficients of a polynomial should be represented in terms of basic material properties, i.e., tensile and compressive strengths in the in terms of similar parameters.

Tsai (1965) has used failure criterion, given by Hill (1948) for orthotropic materials, to describe fallure behavior of unidirectional composites. This 
criterion assumes equal tensile and compressive strengths in a particular direction. A modification of Hill's (1948) criterion has been given by Hof man (1967) and Tsai and Wu (1971) and several other criteria have been proposed. A comprehensive review is given by Owen and Griffiths (1978) where te has been reported that differences in these failure criteria are small and subtle. However, evaluation of polynomial constants is very cumbersome and tedius. Hashin (1980) has recognized this problem recently and has proposed four piece-wise failure criteria to describe four distinct fiber and matrix modes - are modeled separately.
fensile and compressive

Flexural strengths of unidirectional graphite/epoxy and Kevlar/epoxy composites have been measured as a function of orientation angle starting from the iber direction. The measured off-axis flexural strengths are compared with critericted values calculated using maximum stress theory and the failure 1948). Experimental results (1980) and Tsai-Hill (Tsai, 1965, 1968; Hill, ure criterion due to difficulty action term used in the failure eriterion in the evaluation of an inter(1973) have shown in their failure influence of an interaction term on predicted strength values is that the second order.

In this study, off-axis flexural strength tests are used because of simplicity in testing procedure; and unlike tension tests, flexural strength test do not possess end constraints. However, lift-off of the ends tional composite specimen occurs in the three point flexural test (Halpin Pagano, 1969; Whitney and Dauksys, 1970). The end lift-off phenomenon in an erroneous values of mechanical properties obtained using flexural test However, Agarwal and Broutman (1980) have suggested that lift-off of ends is suppressed if a four point flexural test is used in the measurement of offaxis flexural properties. Also, Ishai and Lavengood (1969) have shown experimentally that tensile and flexural off-axis properties of glass-epoxy unidirectional composites are identical if slender specimens having lengththan six and span-to-depth ratio greater than 20 are used (Hoggatt, 1969).

\section{THEORY}

In this section three failure criteria, maximum stress theory (Jones, 1975) Tsai-Hill (Tsai, 1965, 1968; Hill, 1948; Jones, 1975) theory and Hashin's (1980) piece-wise failure criterion, will be discussed to describe failur

\section{Maximum Stress Theory}

This theory predicts that failure will occur as soon as a principal stress in a material exceeds the material strength in that direction. This theory is two dimensional, and it can be applied to analyze effects of two dineni-

If the tensile strengths along $x$ and $y$ are represented by $X$ and $Y$, respectively, $S$ is shear strength in the $x-y$ plane. If a specimen shown in Fig. 1 ned at an angle, $\theta$, from the stress is denoted by $\sigma_{\theta}$.

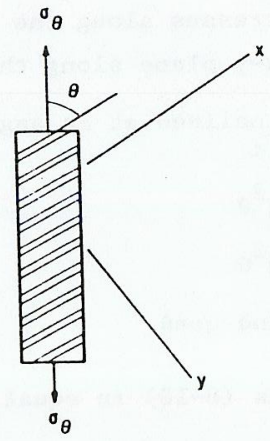

Fig. 1. Off-axis specimen and coordinate system.

Using orthogonal transformation, relationships between external stress and the material constants are as follows:

$$
\begin{aligned}
& X=\sigma_{\theta} \cos ^{2} \theta \\
& Y=\sigma_{\theta} \sin ^{2} \theta \\
& S=\sigma_{\theta} \sin \theta \cos \theta
\end{aligned}
$$
Thus, the above equations relate tensile strength of a unidirectional compos-
ite at an angle, $\theta$, in terms of the strengths of the material in the fiber and transverse to fiber directions and strengths at an angle, $\theta$, are given:

$$
\begin{aligned}
& \sigma_{\theta}=\mathrm{X} / \cos ^{2} \theta \\
& \sigma_{\theta}=\mathrm{Y} / \sin ^{2} \theta \\
& \sigma_{\theta}=\mathrm{S} / \sin \theta \cos \theta
\end{aligned}
$$

As soon as one of the above conditions is met, failure occurs. It can be seen that the above equations are uncoupled, i.e., there is no interaction between the stresses.

\section{Tsai-Hill Theory}

Tsai (1968) used Hill's (1948) criterion, suggested for anisotropic metals, to describe failure behavior of composite materials. For plane stress in the $x-y$ plane, Hill's (1948) criterion reduces to:

$$
\left(\frac{\sigma}{X}\right)^{2}+\left(\frac{\sigma y}{Y}\right)^{2}-\frac{\sigma x^{\sigma} y}{x^{2}}+\left(\frac{\tau x, y}{S}\right)^{2}=1
$$

where: 
$\sigma_{x}$ and $\sigma_{y}$ are stresses along the fiber and transverse to it and $\tau_{x, y}$ is shear stress in the $\mathrm{x}-\mathrm{y}$ plane along the $\mathrm{x}$ axis.

Stresses on a plane inclined at an angle, $\theta$, to the $\mathrm{x}$ axis are given as

$$
\begin{aligned}
& \sigma_{x}=\sigma_{\theta} \cos ^{2} \theta \\
& \sigma_{y}=\sigma_{\theta} \sin ^{2} \theta \\
& \tau_{x y}=\sigma_{\theta} \sin \theta \cos \theta
\end{aligned}
$$

Substituting equations (8-10) in equation (7),

$$
\frac{\cos ^{4} \theta}{\mathrm{X}^{2}}+\left(\frac{1}{\mathrm{~s}^{2}}-\frac{1}{\mathrm{x}^{2}}\right) \cos ^{2} \theta \sin ^{2} \theta+\frac{\sin ^{4} \theta}{\mathrm{Y}^{2}}=\frac{1}{\sigma_{\theta}^{2}}
$$

By knowing material constants, $X, Y$ and $S$, the strength, $\sigma_{\theta}$, of a unidirectional composite at an angle, $\theta$, from the fiber direction can be calculated from equation (11)

\section{Hashin's Piece-wise Failure Criterion}

For the tensile failure mode, Hashin's criteria are given as follows:

Tensile fiber failure mode:

$$
\sigma_{f, \theta}^{2}=\frac{1}{\cos ^{2} \theta\left[\frac{\cos ^{2} \theta}{x^{2}}+\frac{\sin ^{2} \theta}{s^{2}}\right]}
$$

Tensile matrix failure mode

$$
\sigma_{\mathrm{m}, \theta} 2=\frac{1}{\sin ^{2}\left[\frac{\sin ^{2}}{\mathrm{y}^{2}}+\frac{\cos ^{2}}{\mathrm{~s}^{2}}\right]}
$$

where:

$\sigma_{\mathrm{f}}, \theta$ and $\sigma_{\mathrm{m}}, \theta$ are failure strengths of a composite specimen machined at an angle, $\theta$, from the fiber direction and failed in either fiber or matrix mode, respectively. $X$ and $Y$ are tensile strengths in the fiber ( $x$ ) and transverse to fiber (y) directions, respectively. $S$ is the shear strength of a composite in the $x-y$ plane.

It is interesting to note that equations (12) and (13) can be approximated from equation (11) by imposing the following restrictions:

When $\theta$ is small, $\sin ^{4} \theta$ is very small, and it can be neglected. This condition approximates equation (12) when $\theta$ is large, $\cos ^{4} \theta$ is small, and it can be (1980) pientis condition gives equation (13). Figure 2 compares Hashin's for boron/epoxy and glass/epoxy unidirectional composites.

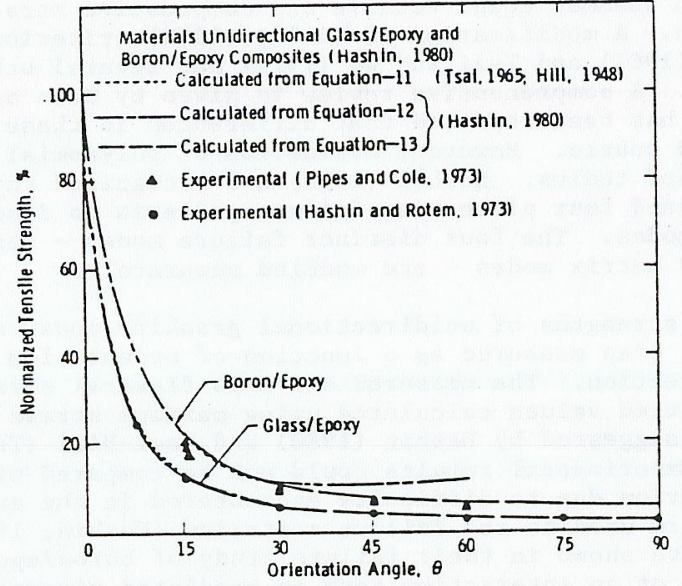

Fig. 2. Comparison of failure theories of Tsai-Hill and Hashin.

tensile off-axis strengths are calculated according to the failure theorie of Hill (1948) and Hashin (1980) using published experimental information (Pipes and Cole, 1973; Hashin and Ratem, 1973). It can be seen that the results obtained from the two theories overlap. Hashin's (1980) theory under plane stress conditions is a special case of Hill's (1948) generalized criterion. Similar conclusions can be drawn when predicting failure behavio of unidirectional composites under plane stress compressive loads.

\section{EXPERIMENTAL}

Graphite/epoxy (Hercules AS4/3501-6) and Kevlar/epoxy (Fiberite HY-E1734A) unidirectional composite prepregs were received. Prepregs were cut to $12 " \mathrm{x} 12$ " size and the plies were stacked unidirectionally in a steel mold to give a 12 " $\times 12$ " $\times 0.2^{\prime \prime}$ thick unidirectional laminate. Laminates were cured at $350^{\circ} \mathrm{F}$ in a hydraulic press following the cure cycle (approximately 50 psi pressure and $350^{\circ} \mathrm{F}$ for 1 hour) recommended by the prepreg manufacturers. It was observed earlier that the unidirectional Kevlar/epoxy laminate is very weak in the transverse direction and testing of the laminate in the transverse direction is very difficult. Thus, it was decided to stack one $90^{\circ} \mathrm{ply}$ each on top and bottom of both the Kevlar/epoxy and graphite/epoxy laminate, respectively, to overcome this problem. These laminates henceforth, will be referred to as cross-ply laminates.

Specimens ( $5 "$ × $1 / 2^{\prime \prime} \times 0.2^{\prime \prime)}$ were machined from unidirectional and cross-ply laminates at a desired angular orientation. Fifteen degree interval for cross-ply laminates and $30^{\circ}$ interval for unidirectional laminates was maintained. Three specimens were tested for each orientation angle in four point flexural test (ASTM D790-71). Average values of flexural strengths are plotted in figures of the following section. 
Figures 3 and 4 give off-axis normalized values of flexural strengths for unidirectional graphite/epoxy and Kevlar/epoxy composite laminates, respec fiver direction.tion of off-axis angle or orientation angle, $\theta$, from the theory, Tsai-Hill (1980) piece-wise (Tsai, 1965, 1968, Hill, 1948; Jones, 1975) and Hashin' experimental infer limited lated values using Equations 8 and than the measured values excet for hied dicted values, assuming shear failure composite (Fig. 4) are in agreement with the this is not true for graphite/epoxy values (Equation 10) are higher than the experiment 3 ) where predicted general, maximum stress theory predicts higher strength values for unidirec tional graphite/epoxy and Kevlar/epoxy composites.

Comparison of plots in Figs. 3 and 4, obtained from Equations 11-13, shows that the Tsai-Hill (Tsai, 1965, 1968; Hill, 1948; Jones, 1975) and Hashin (1980) piece wise failure theories are identical in the different donains. For smaller off-axis angle, $\theta$, values predicted by Equations close together. For higher off-axis angle, $\theta$, Equations 11 and 13 give similar values. The Tsai-Hill (Tsai, 1965, 1968; Hill, 1948; Jones, 1975) theory, unlike the maximum stress theory, and the; Hashin's (1980) piece-w theory, is continuous and does not possess cusps. The Tsai-Hill (Tsai, 1965,

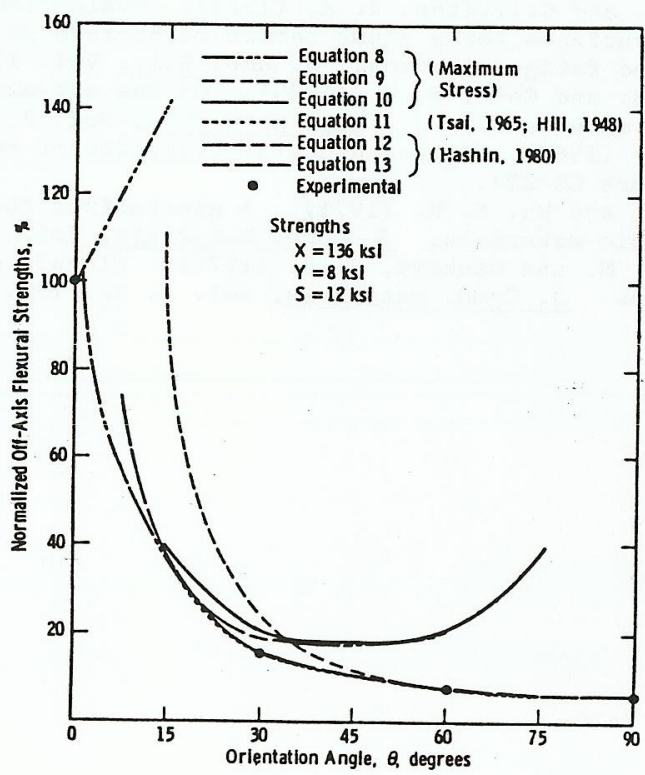

Fig. 3. Normalized off-axis flexural strength versus orientation angle for unidirectional graphite/epoxy composite.

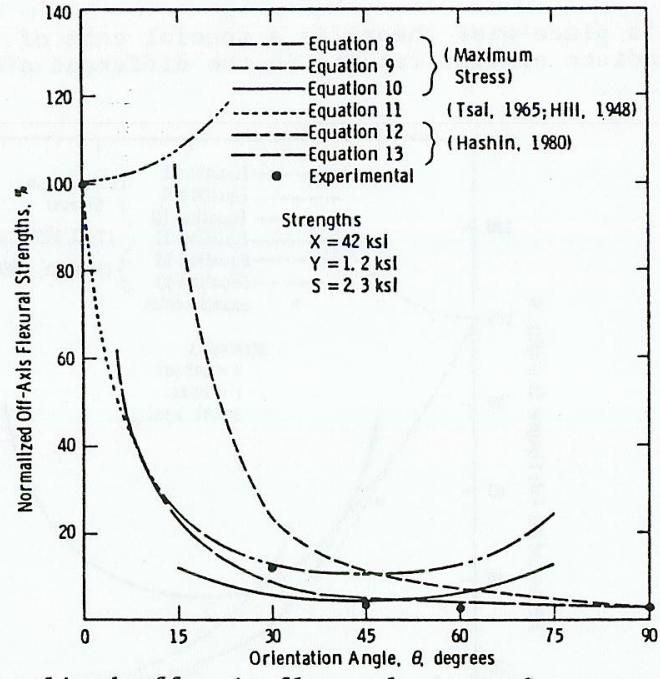
F1g. 4. Normalized off-axis flexural strengths versus orientation

1968; Hill, 1948; Jones, 1975) theory plotted in Figs. 3 and 4 shows close agreement between calculated and experimental off-axis flexural strengths of wintdrectional graphite/epoxy and Kevlar/epoxy composites.

Wormalized flexural strengths for cross-ply $\left[\left(0^{\circ}\right) 20^{90^{\circ}}\right.$ S graphite/epoxy and Revlar/epoxy laminates have been plotted in Figs. 5 and 6 , respectively, as 4 function of off-axis angle. The agreement between calculated values using fad-Hill (Tsai, 1965, 1968; Hi11, 1948; Jones, 1975) calculated values us (1980) piece-wise theory and the off-axis measured values of strengths wery good for graphite/epoxy cross-ply laminates. Discrepancy between is predicted and calculated values is significant for $30^{\circ}$ off-axis angle; reasons for this discrepancy are not clear. However, for Kevlar/epoxy crossfly laminates, the experimental off-axis flexural strength values are lower than the predicted values using three different theories. This discrepancy in be ascribed to the shear failure mode of the cross-ply, i.e., the failure Itd not occur due to the last ply failure in tension or compression; instead It was observed that the failure was due to shearing of the last ply (90 ${ }^{\circ}$ ) trom contiguous $0^{\circ}$ lamina. Also, it has been reported (Deteresa and (o-workers, 1984) that compressive strength of Kevlar fiber is significantly lower than its tensile strength.

\section{CONCLUSIONS}

1. The maximum stress theory, in general, predicts higher off-axis flexural strength values for unidirectional and cross-ply graphite/epoxy and Kevlar/epoxy laminates than the experimental values.

2. Using the Tsai-Hill theory, agreement between calculated off-axis strength values for unidirectional graphite/epoxy, Kevlar/epoxy and cross-ply graphite/epoxy laminate and the experimental results is good. 
3. Hashin's piece-wise theory is a special case of the Tsai-Hill theory eff-axis directions.

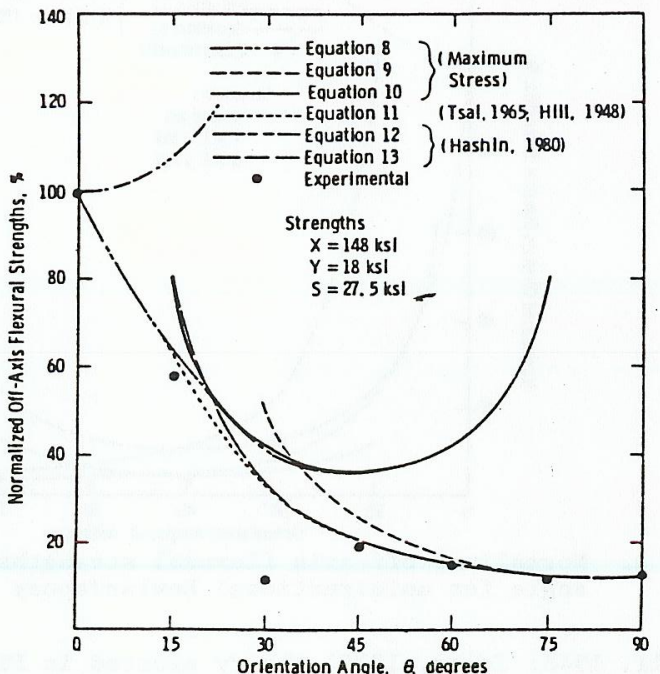

Fig. 5. Off-axis flexural strength versus orientation angle of graphite/epoxy cross-ply $\left[\left(0^{\circ}\right) 20^{90^{\circ}}\right]_{S}$ laminates.

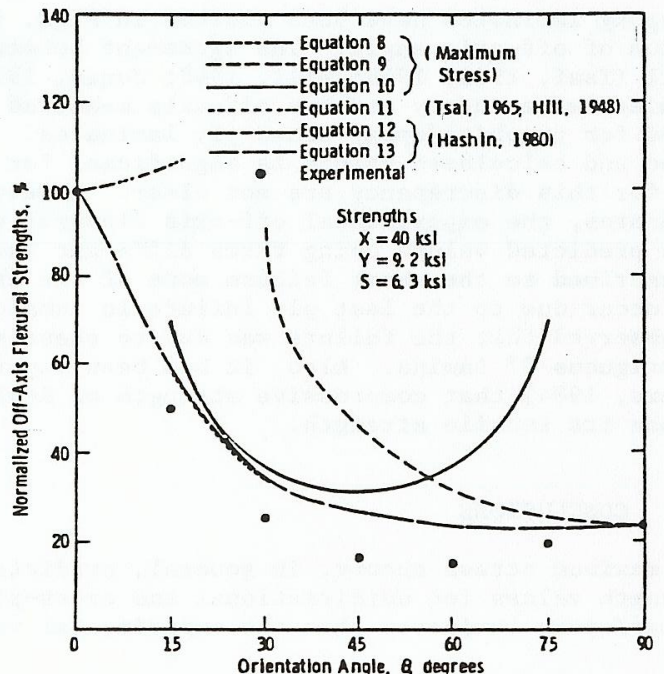

Fig. 6. Normalized off-axis flexural strength versus orientation angle of Kevlar/epoxy $\left[\left(0^{\circ}\right)_{20} 90^{\circ}\right]_{\mathrm{S}}$ laminates.
Agarwal, B. D. and Broutman, L. J. (1980). Analysis and performance of fiber

(leteresa, S. J. Allen, S, R., Farris, J., and Porter, R. S. (1984).

Compressive and torsional behavior of Kevlar 49 fiber. J. Mat. Sci., Vo1. 19, pp. 57.

Ualpin, J. C. Pagana, N. J., Whitney, J. M., and Wu, E. M. (1969).

Characterization of anisotropic composite materials. ASTM Publication: STP-460, Composite Materials: Testing and Design, pp. 37.

Hashin, Z. (1980). Failure criteria for unidirectional fiber composites, J. Appl. Mech., pp. 329

Hashin, Z., and Rotem, A. (1973). A fatigue failure criterion for fiber reinforced materials. J. Comp. Materials, Vol. 7, pp. 448

II11, R (1948). A theory of yielding and plastic flow of anisotropic materials. Proc. Roy. Soc., Series A, Vol. 193, pp. 281.

Hoffman, O. (1967). The brittle strengths of orthotropic materials.

Hoggatt, J. T. (1969). Test methods for high modulus carbon yarn and composites. ASTM Publication: STP-460, Composite Materials: Testing an Design, pp. 48 .

48. Characterizing strength of

( Testing and Design, pp. 271.

, McGraw-liill ones, R. M. (1975)

Book Co., Chapt. Evaluation of biaxial stress failure surfaces for a glass fabric reinforced polyester resin under static and fatigue loading. J. Mat. Sci., Vol. 13, pp. 1521.

static and for anispotrials. J. Comp. Materials, Vol. 7, pp. 246

Tsai, S. W. (1965). Strength characterization of composite materials. NASA Report CR-224.

Tsai, S. W. and Wu, E. M. (1971). A generalized theory of strength for nisotropic materials. J. Comp. Materials, Vol. 5, pp. 58.

Flexure experiments on off-axis composites. J. Comp. Materials, Vo1. 4, pp. 135. 